\title{
HEARING IMPROVEMENT IN PATIENTS WITH COMMUNICATIVE DISORDERS
}

\author{
Alexander Donchev ${ }^{1}$, Mario Milkov² \\ 1) Depatrtment International Cooperation, Medical University Varna, Bulgaria. \\ 2) Faculty of Dental Medicine, Medical University, Varna, Bulgaria.
}

\section{SUMMARY}

For intervention of any communication disorder, prevention is the first and crucial component. Communication disorder is an umbrella term, which includes a variety of speech, language, and hearing disorders. Hearing impairment and hearing loss cause considerable damage to communication skills in children and adults. The communication disorders require timely and proper inverventions by interdisciplinary teams. In the present survey, some effective clinical practices reported in the current world literature are consisely described.

Keywords: communication disorders, hearing impairment, hearing improvement interventions, children, adults,

\section{INTRODUCTION:}

Hearing impairment is one of the communication disorders of the 21 st century constituting a public health issue as it affects communication, academic success, and life quality of students [1]. Most cases of hearing loss before 15 years of age are avoidable, and early detection can help prevent academic delays and minimize other consequences. Recently, the broader implications of hearing loss for the health and functioning of older adults are demonstrated in epidemiologic studies [2]. The findings on the association between hearing loss and poorer health outcomes form the foundation for national initiatives in the USA on hearing loss and public health ranging from the Aging and Cognitive Health Evaluation in Elders clinical trial to the Over-The-Counter Hearing Aid Act.

For intervention of any communication disorder, prevention is the first and crucial component [3]. Communication disorders are hidden disabling conditions that affect the social and emotional well-being, cognition, behaviour [4], academic achievement in the school and college years and vocational choices later in adulthood [5]. Communication disorder is an umbrella term, which includes a variety of speech, language, and hearing disorders [6]. Although several studies describe the communication disorders in a variety of ways, clear and empirically derived definitions and classification systems are lacking [3]. This always results in poorly developed evidence, based on which most identification and management of communication disorder is done across the globe [7].
Social epidemiology of communication disorders and hearing impairment

The analysis of epidemiological and economic data for industrialized countries, North America, and the United States shows that communication disorders are estimated to have a prevalence of $5 \%$ to $10 \%$ [5]. People with communication disorders may be more economically disadvantaged than those with less severe disabilities. Individuals with severe speech disabilities are more unemployed or in a lower economic class than those with hearing loss or other disabilities. Communication disorders may cost the United States from $\$ 154$ billion to $\$ 186$ billion per year, which is equal to $2.5 \%$ to $3 \%$ of the Gross National Product.

The prevalence of speech disorders in 10425 primary school students in Australia is estimated [4]. Stuttering is identified in $0.33 \%$, voice disorder in $0.12 \%$, and speechsound disorder in $1.06 \%$ of the cases. There is a higher prevalence of speech disorders in males than in females. As grade level increases, the prevalence of speech disorders decreases. The students with a speech disorder are more likely to be in the higher socioeconomic groups. There is a difference between the perceived and actual level of support provided to these students.

In an institution-based study, the prevalence rate of auditory impairment among the patients with communication disorders in Minimal, Kartanaka, India, amounts to $62 \%$ $[8]$.

Within a door-to-door survey of 15441 individuals from 15 villages in India, irrespective of their age and gender, prevalence of individuals at risk of communication disorders is $6.07 \%$ [9]. Among those at risk, the prevalence of audiological and/or otological disorders is $90.58 \%$ and that of speech and language ones is $9.42 \%$. Communication disorders are more common in males compared to females. The prevalence of severe and moderately severe hearing loss is higher than other degrees of this disorder.

In Sao Paulo, Brasil, the examinations of speech, language and myofunctional oral aspects of 2980 children aged one to 11 years identify primary communicative disorders in 125 children that represents a prevalence rate of $4.19 \%$ [10]. The most common idiopathic speech-language disorders are articulatory disorders, oral language delays, myofunctional and neurovegetative disturbances. 
Interventions to hearing improvement in adults with communication disorders

Twelve papers describing hearing interventions in adults aged over 50 years with dementia and adult-onset hearing impairment having received a hearing intervention in relation to cognitive function, rate of decline, psychiatric symptoms, hearing-related disability, quality of life, and/or caregiver burden outcomes are retrieved in electronic data-bases and analyzed [11]. One high-quality randomized controlled trial of a hearing aid intervention is identified. Provision of hearing aids, assistive listening devices, communication strategies, hearing aid trouble shooting, and cochlear implantation are included. There is no consistent evidence of the positive impact of hearing interventions on cognitive function, rate of cognitive decline, quality of life, or caregiver burden. Sensory interventions may promote better outcomes, but there is a need for properly powered, controlled trials of hearing interventions on outcomes relevant to people living with dementia.

The multi-step development of a new intervention designed to support hearing function in people living with dementia in their own homes is described [12]. This programme is based on the process of 'intervention mapping' in study centres in Manchester, Nicosia and Bordeaux and comprises four integrated steps, designed to address the following: i) scoping the gaps in understanding, awareness and service provision for the hearing impairment care needs of such people; ii) investigating the support care needs through a literature search, stakeholder surveys, focus groups, semi-structured interviews and an expert reference group; iii) refining the prototype by additional input from stakeholders using focus groups and semi-structured interviews; and iv) field testing the draft intervention using an open-labelled, non-randomized feasibility study, integrating feedback from these people and their significant others to develop the final intervention ready for full scale definitive trialling.

A protocol to field test a newly-developed homebased intervention, designed to optimise sensory functioning in people with dementia in three European sites is analyzed [13]. In a multisite, single arm, open label, feasibility study, 24 participants with dementia are assessed for hearing impairments and prescribed a hearing aid. Each participant has a study partner ('dyads'). A subset of dyads receives 'sensory support' from a 'sensory support therapist', comprising home visits over 12 weeks. The therapist offers the following intervention: adherence support for corrective devices; adaptations to the home environment to facilitate sensory function; communication training; and referral to community-based support services. The primary outcomes are process measures assessing the feasibility, tolerability and acceptability of: i) the intervention components; ii) the method of implementation of the intervention and iii) the study procedures, including outcome assessment measures. This feasibility study is a necessary step in the development of a complex, individualized, psychosocial intervention.

The complex management includes seven components [13]. Component 1 includes identification and cor- rection of any vision or hearing impairment. If needed, hearing aids aer provided. Component 2 represents a continuous training in the correct use of sensory devices. The adherence and correct usage of the prescribed hearing aids is ensured. Component 3 consists in communication training as communication difficulties are a barrier to living well with dementia and sensory impairment. Working with dyads improves communication using adapted, evidence-based materials. Component 4 includes home-based functional assessment and goal setting. Component 5 refers to health and/or social care services in the community as indicated by the assessments and goal-setting exercises. Component 6 provides supplementary sensory devices to enhance the home environment. Component 7 aims at fostering social inclusion through hobbies/interests/social groups.

People with dementia and sensory impairment may become isolated from family and the wider community [14]. It is important to link dyads to community groups and resources based on their hobbies and interests.

Studies on usage of direct-to-consumer hearing devices mainly focus on older adults with hearing loss and report consistently positive results in clinical and self-reported outcome measures [15]. There are improvements in auditory ability, communicative function, social engagement, quality of life, and reduction of self-reported hearing disability.

In a systematic literature review of 13 publications retrieved from CINAHL, MEDLINE, and PsycINFO using EBSCOhost, three categories of direct-to-consumer hearing devices are analyzed: i) personal sound amplification products; ii) direct-mail hearing aids, and iii) over-the-counter hearing aids [16]. Three domains are identified: 1) electroacoustic characteristics, 2) consumer surveys, and 3) outcome evaluations. Some devices meet the stringent acoustic criteria used for hearing aids, while other ones produce dangerous output levels (i.e., over 120-dB sound pressure level). Although direct-mail hearing aids and personal sound amplification products are associated with lower satisfaction when compared to hearing aids purchased through hearing health care professionals, 5\%-19\% of patients with hearing loss purchase hearing aids through direct-mail or online. There are positive outcomes of overthe-counter devices, especially when a hearing health care professional supports these users.

The analysis of a longitudinal sample of 154'783 persons aged $\geq 65$ years in Germany with incident dementia diagnosis and hearing impairment between 2006 and 2010 shows that patients with bilateral (hazard ratio $=1.43$; $\mathrm{p}<0.001$ ) and side-unspecified (hazard ratio $=1.20$; $\mathrm{p}<0.001$ ) hearing impairment have higher risks of dementia incidence than patients without this disorder [14]. The effect of hearing impairment is only partly mediated through otorhinolaryngologist's utilization. Significant interaction between hearing impairment and specialist care, care level, and institutionalization, respectively, indicates moderating effects. The importance of the association between hearing impairment and dementia is underlined.

The benefit of having preserved acoustic hearing in the implanted ear for speech recognition in complex lis- 
tening environments is assessed in 21 English-speaking and 17 Polish-speaking cochlear implant recipients under different conditions [17]. There are small, but significant, improvements in performance (1.7-2.1 dB and 6-10 percentage points) for the best-aided condition versus the bimodal one. Postoperative thresholds in the implanted ear correlate with the degree of electric and acoustic stimulation benefit for speech recognition in diffuse noise. There is a significant correlation between interaural time difference threshold at $250 \mathrm{~Hz}$ and electric and acoustic stimulationrelated benefit for the adaptive speech reception threshold. The preserved low-frequency hearing improves speech understanding for cochlear implant recipients.

The effectiveness of a recently developed, lowcost, 'over-the-counter' style hearing aid in 19 elderly patient with mild to moderate mixed or sensorineural hearing loss during a three-month period is evaluated [18]. Three questionnaires (the Client-Oriented Scale of Improvement, the International Outcome Inventory for Hearing Aids and the Profile of Hearing Aid Performance - Chinese version) are completed and an open-ended interview is conducted. This hearing aid provides appropriate amplification and benefitd from improved communicative abilities for these patients as 16 of the patients ( $84.21 \%$ of the cases) use their hearing aid from at least one to over eight hours each day. All the patients consider the low-cost instrument 'worth the trouble' of wearing. While wearing their hearing aids, the patients experience difficulties only infrequently in most everyday listening situations.

In speech-in-noise listening experiments involving 21 young adult listeners, speech samples by older adults with normal hearing and older adults with hearing loss are rated and perceived as less intelligible than those of young subjects [19]. The presence of presbycusis affects the clear speech strategies adopted by older adults with hearing loss talkers and task effectiveness. They show some adaptations consistent with an increase in vocal effort, and it takes them significantly longer than the young subjectsto complete the diapix task. The relative energy in the $1-3 / \mathrm{kHz}$ frequency region of the long-term average spectrum best predicts the intelligibility of speech samples and task transaction time in simulated hearing loss status. Even mild presbycusis in 'healthy aged' adults can affect the dynamic adaptations in speech beneficial for effective communication.

Interventions to hearing improvement in children with communication disorder

Medical care should be provided to communicationdisabled children by otolaryngologists in cooperation with speech language hearing therapists [20]. Examination of infants by speech language hearing therapists enables early detection of variously disabled children, and thereby makes it possible to initiate necessary care quickly. A significant improvement of treatment quality is a major benefit of such collaboration.

The growth of early vocabulary and its composition during the first year after cochlear implant activation is examined in 20 children ( 12 boys and eight girls at a mean age at cochlear implant activation of 12.9 months) [21].
Vocabulary size is assessed with the Finnish version of the MacArthur Communicative Development Inventories Infant Form and compared with normative data. Vocabulary composition is analysed in relation to vocabulary size. Despite clear vocabulary growth over time, children with bilateral cochlear implants lag behind their age norms in receptive vocabulary during the first 12 months after cochlear implant activation. In expressive vocabulary, $35 \%$ of the children are able to catch up with their age norms while $55 \%$ of the children lag behind them. In receptive and expressive vocabularies of 1-20 words, analysis of different semantic categories indicates that social terms constitute the highest proportion. There is a significant main effect of time, gender, maternal education and residual hearing with hearing aids before implantation on early receptive vocabulary growth. Time and residual hearing with hearing aids has a significant main effect on expressive vocabulary growth, too.In four children who have received their first cochlear implant under 12 months of age, the effects on auditory development are determined using the German LittlEARS Auditory Questionnaire, closed- and open-set monosyllabic word tests, aided free-field, the Mainzer and Göttinger speech discrimination tests, Monosyllabic-Trochee-Polysyllabic test, and Listening Progress Profile test [22]. These children reach open-set monosyllabic word discrimination at an age of 24 months. Listening Progress Profile test results improve over time, and children recognize $100 \%$ of words in the Monosyllabic-Trochee-Polysyllabic test after 12 months. All children perform as well as or better than their hearing peers in speech production and grammar development.During a longitudinal and comparative study of 36 children with cochlear implants and 19 children with hearing aids/bone anchored hearing aids in Denmark, a three-year Nordic Auditory Verbal intervention is implemented [23]. Their median age at intervention is 13 and 12 months, respectively. There is a significant difference between children with hearing impairment regardless of hearing technology and children with normal hearing. Although children with hearing impairment progress over a three-year period, they do not reach the same level as children with normal hearing. The impact of the current Early Hearing Detection and Intervention policy on vocabulary outcomes in 448 children with bilateral hearing loss between 8 and 39 months $(25.3 \pm 7.5$ months $)$ of age is examined during a cross-sectional study [24]. These children live in 12 different states and participate in the National Early Childhood Assessment Project. Vocabulary quotients are significantly higher for children who met the Early Hearing Detection and Intervention guidelines, are younger, have no additional disabilities, mild to moderate hearing loss as well as which parents are deaf or hard of hearing and which mothers are with higher education levels. Audiological/language test scores and demographic factors are compared among 210 prelingual severe-to-profound hearing-impaired children with cochlear implants from the Research on Sensory and Communicative Disorders project in Japan to identify prognostic factors for language development [25]. Of them, 49 children have received first hearing aid before the age of six months while the rest 161 ones 
do it after the age of seven months. The children in the first group present with statistically significantly higher scores on comprehensive vocabulary and academic achievement $(\mathrm{p}<0.05)$. There are no significanrt differences between 71 children who have received newborn hearing screening and 129 ones without this examnination although the latter are statistically significantly older and have used cochlear implants longer $(\mathrm{p}<0.05)$. A new use of video analysis in deaf children with associated disabilities and communication disorders is proposed [26]. The set-up of the recording is the same as the standard method and 20 communication turns are evaluated. For deaf children with complex needs, it is very important to add to the standard parameters the analysis of the maternal communicative style. The video is a useful evaluation tool in deaf children with associated disabilities even if not applied in its standard form. The effect of two interventions to improve the communicative and social skills of four preschoolers with hearing loss in two learning environments, i.e. a preschool classroom for deaf children (oral approach) and an inclusive regular preschool classroom is examined [27]. In the inclusive settings, three participants increase verbal comments although some changes are slight. In the oral preschool classroom, two children show improvements in target behaviours with both interventions. Generalization of skills occurs in two participants. A six-year-old boy loses his normally-developed language ability within two months after bilateral sudden peripheral deafness [28]. The boy becomes non-communicative with others, restless with frequent meaningless bursts of shouts. These communication skills are equivalent to 9-11 months of language development and indicate a rapid breakdown of language). Since conservative methods are ineffective, cochlear implantation is performed. There is a surprising success as he regains the language retracing the normal developmental stages and catches up with his contemporaries in two years. Behavioural improvement parallels his language development.

In conclusion, hearing improvement of children and adults with communication disorders is a complex interdisciplinary task which proper solution deserves much more attention by the medical and psychological scientific community worldwide.

\section{REFERENCES:}

1. Nunes ADDS, Silva CRL, Balen SA, Souza DLB, Barbosa IR. Prevalence of hearing impairment and associated factors in school-aged children and adolescents: a systematic review. Braz J Otorhinolaryngol. 2018 Dec 1. pi i : S 1808 - 8694 ( 18 ) 30321 - 5 . [PubMed] [Crossref].

2. Goman AM, Lin FR. Hearing loss in older adults - From epidemiological insights to national initiatives. Hear Res. 2018 Nov;369:29-32.

3. Sinha SK, Shivaswamy J, Barman A, Seth D, Seshadri D, Savithri SR. Prevalence of communication disorders in a rural population at taluq level of Gujarat, India. Clin Epidemiol Global Hlth. 2017;5(2):73-78.

4. McKinnon DH, McLeod S, Reilly S. The prevalence of stuttering, voice, and speech-sound disorders in primary school students in Australia. Lang Speech Hear Serv Sch. 2007 Jan;38(1):5-15.

5. Ruben RJ. Redefining the survival of the fittest: communication disorders in the $21^{\text {st }}$ century. Laryngoscope. $2000 \mathrm{Feb} ; 110(2 \mathrm{Pt}$ 1):241-245.

6. Ruscello DM, St Louis KO, Mason N. School-aged children with phonologic disorders: coexistence with other speech/language disorders. J Speech Hear Res. 1991;
34(2):236-242.

7. Reilly S, Douglas J, Oates J (eds.). Evidence based practice in speech pathology. London: Whurr; 2004.

8. Devadiga D, Varghese AL, Bhat J. Epidemiology of communication disorders and its role in rehabilitation. Int J Innov Res Dev. 2014;3(13):469473.

9. Konadath S, Suma C, Jayaram G, Sandeep M, Mahima G, Shreyank PS. Prevalence of communication disorders in a rural population of India. $J$ Hear Sci. 2013 Jun;3(2):41-49.

10. de Andrade CRF. Prevalência das desordens idiopáticas da fala e da linguagem em crianças de um a onze anos de idade (Speech-language idiopathic disorder prevalence in children from one to eleven years of age). Rev Saude Publ. 1997;31(5):495-501.

11. Dawes P, Wolski L, Himmelsbach I, Regan J, Leroi I. Interventions for hearing and vision impairment to improve outcomes for people with dementia: a scoping review. Int Psychogeriatr. 2018 Sep 24:1-19. [PubMed] [Crossref]

12. Leroi I, Pye A, Armitage CJ, Charalambous AP, Constantinidou F, Helmer C, et al. Research protocol for a complex intervention to support hearing and vision function to improve the lives of people with dementia. $P i$ lot Feasibility Stud. 2017 Sep 11;3:38. [PubMed] [Crossref]

13. Regan J, Dawes P, Pye A, Armitage CJ, Hann M, Himmelsbach I, et al. Improving hearing and vision in dementia: protocol for a field trial of a new intervention. BMJ Open. 2017 Nov 28;7(11):e018744. [PubMed] [Crossref]

14. Fritze T, Teipel S, Óvári A, Kilimann I, Witt G, Doblhammer G. Hearing impairment affects dementia incidence. An analysis based on longitudinal health claims data in Germany. PLoS One. 2016 Jul 8;11(7): e0156876. [PubMed] [Crossref]

15. Tran NR, Manchaiah V. Outcomes of direct-to-consumer hearing devices for people with hearing koss: a review. J Audiol Otol. 2018 Oct; 22(4):178-188.

16. Manchaiah V, Taylor B, Dockens AL, Tran NR, Lane K, Castle $\mathrm{M}$, et al. Applications of direct-to-consumer hearing devices for adults with hearing loss: a review. Clin Interv Aging. 2017 May 18;12:859-871.

17. McPherson B, Wong ET. Effectiveness of an affordable hearing aid with elderly persons. Disabil Rehabil. 2005 Jun 3;27(11):601-609. 
18. Hazan V, Tuomainen O, Tu L, Kim J, Davis C, Brungart D, et al. How do aging and age-related hearing loss affect the ability to communicate effectively in challenging communicative conditions? Hear Res. 2018 Nov;369:33-41.

19. Udaka J, Kasai SI. Collaboration between otolaryngologist and speech language hearing therapist in the ENT clinic. Jap J Logoped Phoniatr. 2017;58(4):310-316.

20. Välimaa T, Kunnari $\mathrm{S}$, Laukkanen-Nevala P, Lonka E; National Clinical Research Team. Early vocabulary development in children with bilateral cochlear implants. Int $J$ Lang Commun Disord. 2018 Jan; 53(1):3-15.

21. May-Mederake B, ShehataDieler W. A case study assessing the auditory and speech development of four children implanted with cochlear implants by the chronological age of 12 months. Case Rep Otolaryngol. 2013; 2013:359218. [PubMed]

\section{[Crossref]}

22. Percy-Smith L, Hallstrøm M, Josvassen JL, Mikkelsen JH, Nissen L, Dieleman E, et al. Differences and similarities in early vocabulary development between children with hearing aids and children with cochlear implant enrolled in 3-year auditory verbal intervention. Int $J$ Pediatr Otorhinolaryngol. 2018 May;108:6772.

23. Yoshinaga-Itano C, Sedey AL, Wiggin M, Chung W. Early hearing detection and vocabulary of children with hearing loss. Pediatrics. 2017 Aug;140(2). doi: 10.1542/peds.20162964.

24. Sugaya A, Fukushima K, Kasai N, Kataoka Y, Maeda Y, Nagayasu R, et al. Impact of early intervention on comprehensive language and academic achievement in Japanese hearing-impaired children with cochlear implants. Int J Pediatr Otorhinolaryngol. 2015 Dec;79(12): 2142-2146.

25. Ghiselli S, Montino S. Improve- ment of the video analysis method for the evaluation of communication skills in deaf children with complex needs. Hear Balance Commun. 2016;14(2):70-76.

26. Gifford RH, Dorman MF, Skarzynski H, Lorens A, Polak M, Driscoll CL, et al. Cochlear implantation with hearing preservation yields significant benefit for speech recognition in complex listening environments. Ear Hear. 2013 Jul-Aug;34(4): 413-425.

27. Raver SA, Bobzien J, Richels C, Hester P, Anthony N. Using dyad-specific social stories to increase communicative and social skills of preschoolers with hearing loss in self-contained and inclusive settings. Int $J$ Inclus Educ. 2014;18(1):18-35.

28. Ito K, Suzuki Y, Toma M, Shiroma M, Kaga K. Postlingual collapse of language and its recovery after cochlear implantation. Int J Pediatr Otorhinolaryngol. 2002 Feb 25;62(3): 261-265.

Please cite this article as: Donchev A, Milkov M. Hearing improvement in patients with communicative disorders. $J$ of IMAB. 2019 Apr-Jun;25(2):2476-2480. DOI: https://doi.org/10.5272/jimab.2019252.2476

\section{Address for correspondence:}

Assoc. Prof. Mario Milkov, MD, PhD.

Department of Dental Materials Science and Propaedeutics of Prosthetic Dental Medicine, Faculty of Dental Medicine, Medical University Prof. Paraskev Stoyanov of Varna

55, Marin Drinov Str., 9002 Varna, Bulgaria

E-mail: mariomilkov@gmail.com 